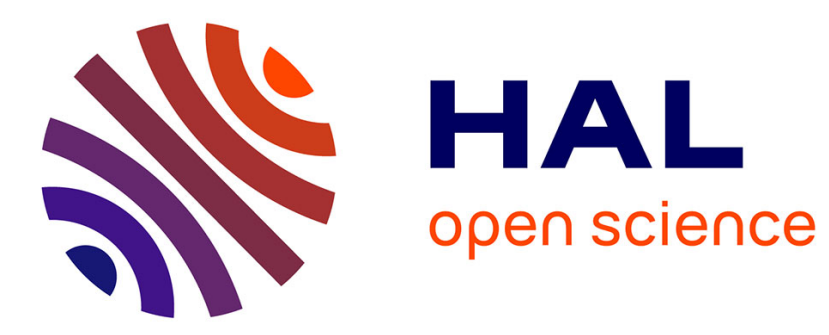

\title{
Numerical assessment of fan noise confinement effects in a closed wind-tunnel
}

Mathieu Lorteau, Thomas Le Garrec

\section{To cite this version:}

Mathieu Lorteau, Thomas Le Garrec. Numerical assessment of fan noise confinement effects in a closed wind-tunnel. AIAA AVIATION Forum, Aug 2021, Virtuel, United States. hal-03378419

\section{HAL Id: hal-03378419 \\ https://hal.science/hal-03378419}

Submitted on 14 Oct 2021

HAL is a multi-disciplinary open access archive for the deposit and dissemination of scientific research documents, whether they are published or not. The documents may come from teaching and research institutions in France or abroad, or from public or private research centers.
L'archive ouverte pluridisciplinaire HAL, est destinée au dépôt et à la diffusion de documents scientifiques de niveau recherche, publiés ou non, émanant des établissements d'enseignement et de recherche français ou étrangers, des laboratoires publics ou privés. 


\title{
Numerical assessment of fan noise confinement effects in a closed wind-tunnel
}

\author{
Mathieu Lorteau* and Thomas Le Garrec ${ }^{\dagger}$ \\ Aerodynamics, Aeroelasticity and Acoustics Department, \\ ONERA - The French Aerospace Lab, \\ 29 avenue de la Division Leclerc, 92320 Châtillon, France
}

\begin{abstract}
This work investigates, through numerical simulations, the acoustic confinement effects of a modern turbofan noise bench installed in the ONERA S1MA wind-tunnel with a closed test section at low Mach number equipped with a dedicated acoustic liner. These simulations aim at preparing acoustic experimental measurements of the D13 UHBR engine demonstrator SA2FIR (Simulator of Aerodynamic and Acoustic Fan IntegRation) of the Clean Sky 2 LPA IADP (Large Passenger Aircraft - Innovative Aircraft Demonstration Platform) and more precisely in the workpackage WP1.1.12 of the ADEC (ADvanced Engine and aircraft Configurations) project.

The present activity makes use of a full immersed boundary workflow developed in previous works, which consists in computing with a CAA solver, the propagation of the acoustic waves generated by analytic fan/OGV interaction modes injected in the nacelle duct, in the presence of complex geometries and a realistic non-uniform mean flow previously obtained with a CFD solver. Both CFD and CAA solvers use the Immersed Boundary Method to deal with the bench and wind tunnel geometries, which greatly simplifies the meshing stage and also drastically reduces the overall computational time.

The confined configuration with lined walls is compared to an isolated nacelle configuration without nacelle support, in terms of acoustic levels and directivity diagrams. Comparisons also include two additional simulations: confined set-up with rigid walls and isolated nacelle with its support to complete the results. The numerical simulations demonstrate the ability of the lined facility to recover the radiated acoustic field of the target free-field configuration.
\end{abstract}

\section{Introduction}

An important key point to reduce aircraft noise consists in the understanding of the physical mechanisms behind the noise generation process. In this context, it is necessary to have access to reliable experimental database, in particular on turbofan noise which constitutes nowadays a growing acoustic source of an aircraft. Modern UHBR (Ultra High Bypass Ratio) aeroengines are characterized by reduced fan speeds and very short asymmetric intakes, so that the mean flow entering into the nacelle is no more uniform along the azimuthal direction and can modify the tonal noise characteristics known from conventional turbofans. To help in the understanding of these mechanisms, the D13 UHBR engine demonstrator SA2FIR is developed within the framework of the Clean Sky 2 LPA IADP platform. Within this platform, the workpackage WP1.1.12 of the ADEC project, in particular, aims to prepare acoustic experimental measurements in an aerodynamic closed wind-tunnel, with acoustic liners, which constitutes a key-point to have access to reliable database.

This study focuses on the fan noise confinement effects in the ONERA S1MA wind-tunnel. To this aim, the methodology first implemented by Mincu et al. [1] and further improved by Lorteau et al. [2] is used, the latter authors studied fan noise installation effects considering a realistic aircraft geometry using the Immersed Boundary Method (IBM). This method is particularly straightforward to take into account the effects of, not only the solid walls, but other boundary conditions and thus enables to overcome the mesh design difficulties inherent to complex geometries.

In the present work, the IBM workflow implemented in the CAA solver $s A b r i n A \_v 0$ [3-7] is used to study the confinement effects in a closed test-section, equipped with acoustic absorbent material, of the noise radiated by a turbofan bench with a realistic geometry in presence of a realistic mean flow at a Mach number $M \sim 0.25$ corresponding to take-off / landing flight conditions. To this aim, the radiated acoustic field from the same turbofan bench is compared

\footnotetext{
*Research engineer, mathieu.lorteau@onera.fr

${ }^{\dagger}$ Research engineer, thomas.le_garrec@onera.fr
} 
in the confined and in free-field configurations. Firstly, a realistic mean flow is computed around the geometry under consideration. Secondly, this mean flow is interpolated on the CAA grid and used afterwards in the CAA computation in which a simplified fan/OGV interaction noise model is considered to enforce the acoustic source inside the duct.

This paper is organized as follows. In section III, the methodology used to evaluate the acoustic installation effects is briefly reminded, some details are given on both the CFD and the CAA computations. Then, section III presents the numerical parameters used in this work: the configuration of interest as well as the meshes (CFD and CAA) on which the simulations were performed. In section IV, the nearfield acoustics and sound pressure levels on antennae are presented and analysed. The main conclusions and perspectives of this work are given in section $\mathrm{V}$

\section{Numerical methodology}

To study the acoustic confinement effects of a turbofan in a wind-tunnel, we intend to compute the noise radiated by this turbofan taking into account the wind-tunnel geometry and the mean flow effects. To this aim, the full-Immersed Boundary methodology detailed in Mincu et al. [1] and Lorteau et al. [2] is used, so only a brief summary is given in this section. A two-step methodology is used: CFD then CAA. Firstly, a realistic mean flow is obtained by a RANS simulation around the geometry under consideration, the geometry being taken into account via the IBM and the mesh being a multi-block structured Cartesian grid. In a second step, this mean flow field is interpolated from the CFD grid to the CAA grid by the use of the in-house Cassiopée tool [8]. As mentioned in section I] in the CAA computation, the geometry of interest is also taken into account with the IBM approach, which thus greatly simplifies the CAA mesh design process for such a geometry, the mesh simply consisting in a single 3D block Cartesian grid. Then, the CAA computation is performed to propagate an acoustic source model on the mean flow. For potential propagation over long distance, an additional step can be added, consisting in propagating the near field into the far field acoustics using a Kirchhoff integral method[9, 10], to compute acoustic maps for instance.

Thus, the present methodology uses a full-IBM workflow on Cartesian grids, which greatly simplifies the mesh generation process: only a triangular surface mesh is necessary to define the geometry and a Cartesian mesh to compute either the flow field or the acoustic field. Moreover, this workflow allows to maximize the explicit time step of the CAA computation and thus to drastically reduce the computational time. All these numerical components make the proposed methodology very efficient to study acoustic installation effects over complex geometries. More information on the solvers used for the CFD and the CAA steps are provided in the following sections.

\section{Numerical parameters}

\section{A. Nacelle and test section geometries and operating condition}

The nacelle considered for the SA2FIR test bench includes an asymmetric intake with a small droop and a pylon as illustrated in figure 1. The fan stage is issued from the ASPIRE project [11, 12] and has been scaled giving a nacelle radius around $0.3 \mathrm{~m}$. As can be seen in figure 1. b), no fan stage (rotor \& stator) is included, since for the CAA computations analytical acoustic sources have been considered. However two internal deviations at 0 and 6 o'clock are taken into account in the simulations to connect the hub to the nacelle, one of which serves also to attach the pylon to the nacelle.

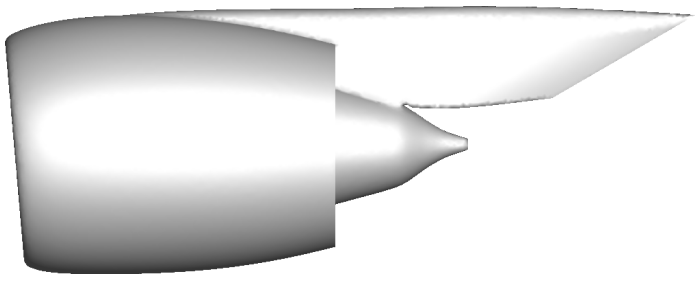

(a)

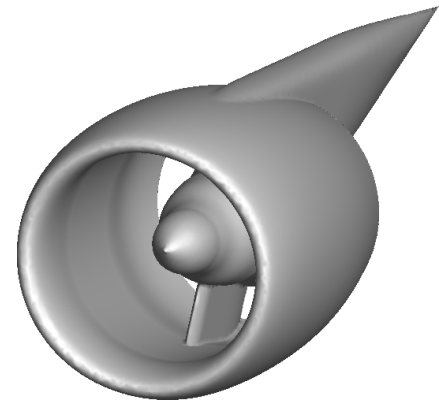

(b)

Fig. 1 Illustrations of the SA2FIR nacelle in (a) the $z=0$ plane and (b) a global view 
The wind-tunnel considered is the ONERA S1MA facility[13-16] in which the SA2FIR test bench is going to be experimentally tested. The test section, illustrated in figure 2 , is $14 \mathrm{~m}$ long in the flow direction and its diameter is about $8 \mathrm{~m}$. The nacelle is located a little below the wind-tunnel test section axis for setup considerations. Moreover, in order to damp the direct acoustic waves emitted from the nacelle, dedicated acoustic foam panels can equip all the test section walls for tests up to Mach number $M=0.3$. The figure 2 represents the SA2FIR turbofan test bench installed in the S1MA test section equipped with the acoustic foam panels, the flow comes from the left-hand side of the figure. As can be seen, the test bench is linked to the wind-tunnel floor by a support with a streamlined shape in the continuity of the nacelle's hub. The pylon of the nacelle and the arm support are rotated by a $90^{\circ}$ angle, this favours the measurements in the flyover direction, as well as in the sideline directions. By doing so, the nacelle support is not in the flyover plane and the distance between the nacelle and the wind-tunnel walls is maximized. As previously mentioned, two CAA computations have been performed in the confined configuration. Both computations have been performed with the lined wind-tunnel geometry, the only difference coming from the boundary conditions being "rigid wall" or "impedance" boundary conditions.

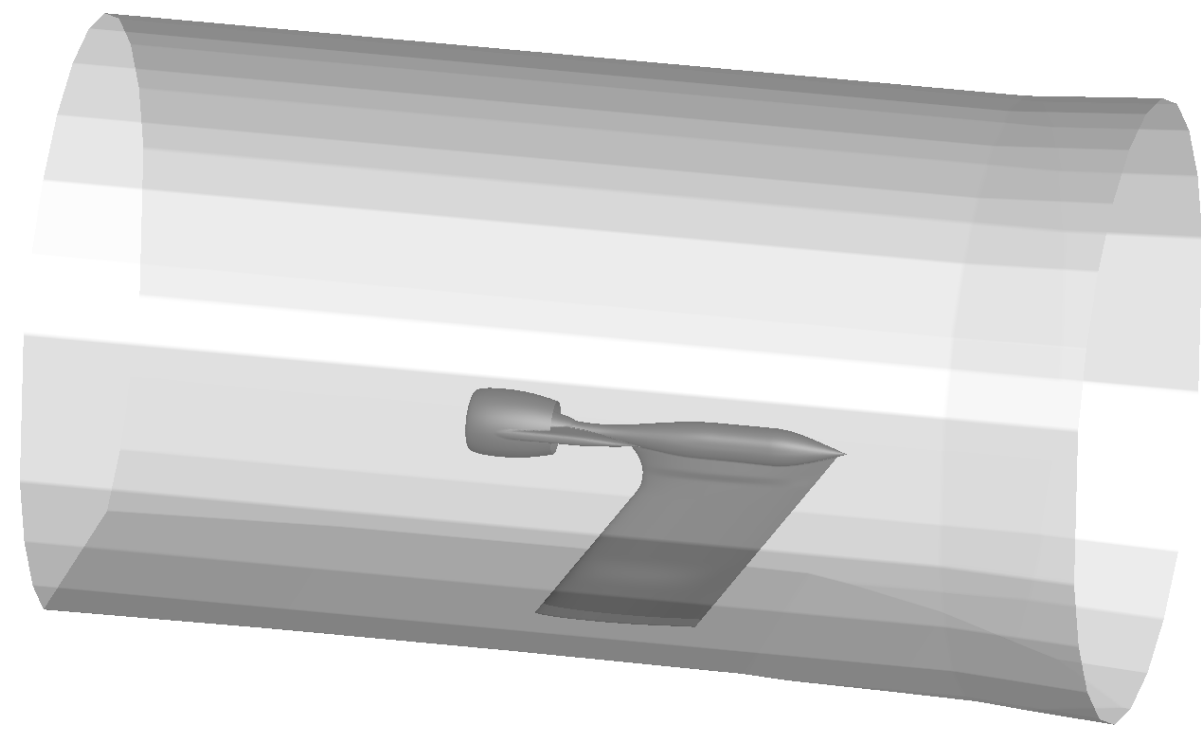

Fig. 2 Illustration of the SA2FIR nacelle with the nacelle support in the wind-tunnel test section

The ASPIRE fan stage has a cut-off design for the BPF 1 (Blade Passing Frequency) with $B=16$ rotor blades and $V=36$ OGV's. The target bypass ratio is equal to 16 and the in-flight minimum thrust was chosen to be representative of a modern mid-sized passenger aircraft. The main turbofan characteristics and operating conditions for the approach case are summarized in the table 1 . For this study, no angle of attack was considered.

\begin{tabular}{ccccc}
\hline \hline Blade \# & Vane \# & Bypass ratio & Fan rotation speed & Flight Mach number \\
\hline 16 & 36 & 16 & $56 \%$ & 0.23 \\
\hline \hline
\end{tabular}

Table 1 Turbofan characteristics and operating conditions for the approach case

Four acoustic simulations are performed and their main characteristics are summarized in table 2 The "FF" set-up corresponds to the ideal case (no set-up effects due to the nacelle support nor confinement effects) and will thus serve as a reference in the following. The "WT_lined " simulation is representative of the realistic experimental set-up, i.e. inside a closed wind-tunnel test section, with a nacelle support and lined walls to damp the acoustic waves and prevent the acoustic reflections, and thus will be evaluated against the "FF" simulation. The two other simulations "FF_support" and "WT_rigid" correspond to two extreme cases for experimental acoustic measurements: without any reflections and with maximum reflections. They will serve as comparisons with the "WT_lined" simulation to evaluate its quality. The two flow fields "free-field" and "confined" are detailed in the next section. 


\begin{tabular}{lccccc}
\hline \hline Case name & nacelle & support & WT walls & liner & mean flow \\
\hline FF & $\times$ & $\varnothing$ & $\varnothing$ & $\varnothing$ & free-field \\
FF_support & $\times$ & $\times$ & $\varnothing$ & $\varnothing$ & confined \\
WT_rigid & $\times$ & $\times$ & $\times$ & $\varnothing$ & confined \\
WT_lined & $\times$ & $\times$ & $\times$ & $\times$ & confined \\
\hline \hline
\end{tabular}

Table 2 Characteristics of the acoustic simulated configurations $(\times$ included or $\varnothing$ not)

\section{B. Mean flow computation}

Two mean flow fields are mandatory: one for the isolated nacelle without its support ("free-field" in table 2), the other for the nacelle and its support inside the wind-tunnel ("confined" in table 2). Similarly to Lorteau et al. [2], the mean flow around the geometry is computed via a RANS approach using the Cartesian solver of FastS [17- 22] developped at ONERA, the geometry being taken into account via the IBM. More details on the IBM implemented in Fast $S$ can be found in Péron et al. [18, 21].

For both simulations, the fan action is modelled by two disks, one upstream and the other one downstream of the fan. The two disks are depicted by the hole inside the nacelle visible in figure 3 (a). On the upstream disk (i.e. close to the nacelle intake), an outlet boundary condition based on a uniform static pressure $p_{\text {out }}$ is imposed while on the downstream disk, an injection boundary condition based on uniform stagnation variables $\left(p_{i}, H_{i}\right)$ is imposed. The two boundary conditions are independent from each other, thus different boundary conditions parameters values had to be tested in order to get a similar mass flow rates on both sides of the fan and to reach the target point. To compute the flow field inside the wind-tunnel as well as the boundary layer developing at the wall, similar boundary conditions are imposed at the inlet and outlet sections of the wind-tunnel in addition to those for the nacelle flow.

The CFD meshes are composed of $55 \times 10^{6}$ cells with 560 Cartesian blocks for the isolated nacelle computation and $36 \times 10^{6}$ cells with 551 Cartesian blocks for the confined configuration. A supplementary mesh refinement was necessary for the isolated nacelle computation for convergence purpose of the jet flow. Thanks to the nacelle support, this refinement was not necessary for the confined configuration. Longitudinal cuts in a mid-nacelle plane of the 3D mesh are depicted in figure 3. where the local mesh refinements near the sharp edges of the geometry can be noticed.

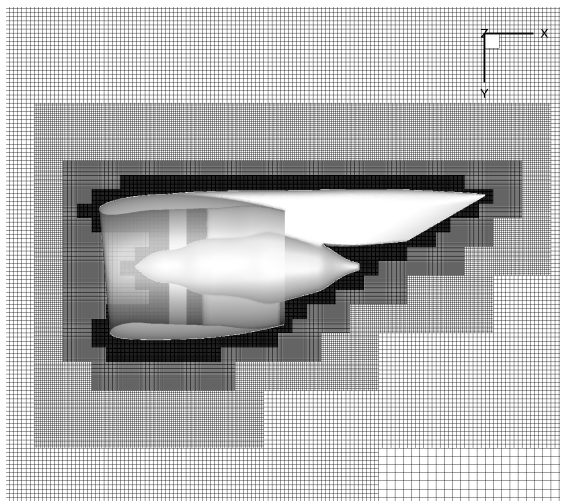

(a)

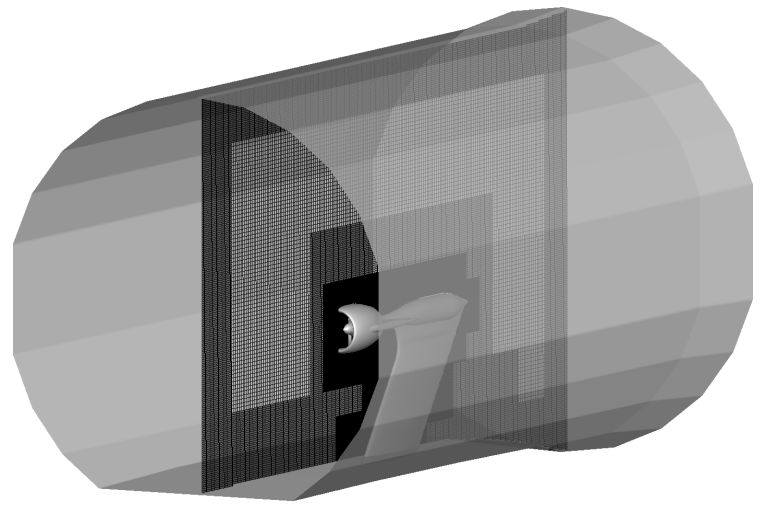

(b)

Fig. 3 Illustration of the CFD mesh for the two mean flow computations: (a) around the isolated nacelle, (b) for the nacelle and its support inside the wind-tunnel

The turbulence modelling is handled by the Spalart-Allmaras model [23]. Musker's algebraic wall function is applied within the IBM approach on Cartesian grids to solve high-Reynolds number flows. The second-order accurate Roe-MUSCL spatial scheme is used with a first-order accurate implicit time integration and a local time step. The RANS computation was performed on 1 node of an ONERA in-house cluster with 24 OMP threads. More than $15 \times 10^{3}$ iterations were computed to ensure a convergence of the residuals (a decrease of about 3 orders of magnitude was obtained). The total computational time was about 350 hours (elapsed) for each computation.

These simulations aim at getting a realistic mean flow on which a realistic propagation of acoustic waves emitted from the nacelle can be computed. These simulations were performed thanks to the experience developed at ONERA 
for the simulation of flows on similar configurations [21, 22]. Thus the simulated flow fields can be considered quite realistic, notably concerning the boundary layer on the wind-tunnel walls. Yet, the aim of the study is not focused on obtaining a mean flow, that is why we will not expand on the flow validation. Color maps of the mean flow around and inside the nacelle are plotted in figure 4. Some slight discrepancies between the two flow fields can be observed in figures 4 (a) and (c), for instance, the isolated nacelle configuration presents a Mach number $M=0.23$ whereas for the confined configuration it is $M=0.25$. This is related to the different boundary conditions which generated the flow around the nacelle (reference state for the isolated nacelle configuration versus injection+outlet for the confined configuration). Both flows are similar at the nacelle intake and present a small distortion effect due to the drooped inlet.

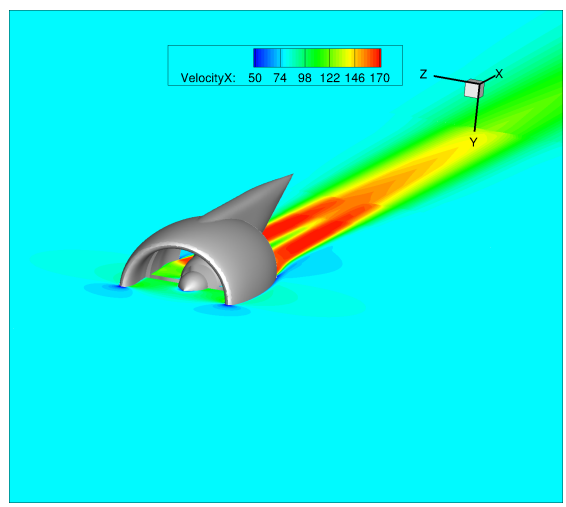

(a)

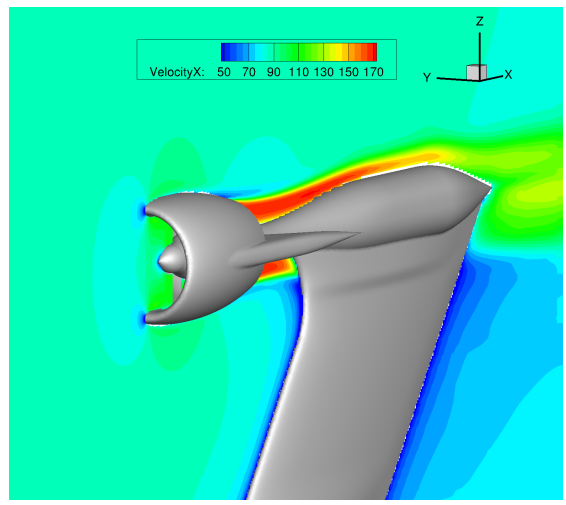

(c)

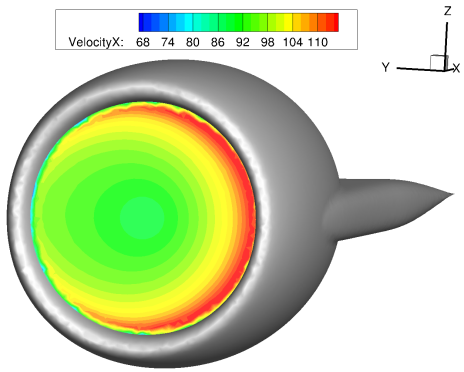

(b)

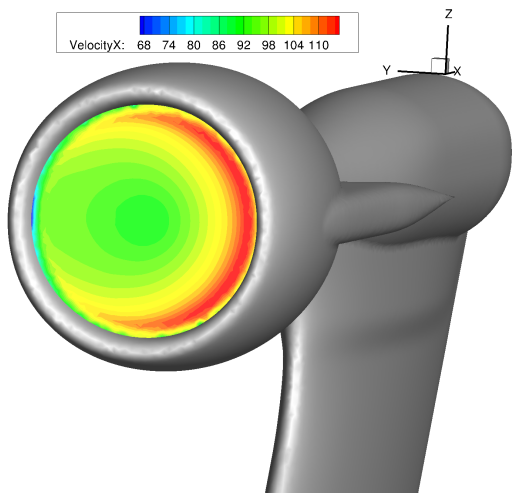

(d)

Fig. 4 Contour maps of axial mean velocity of the overall flow (left) with cut views at the intake highlight plane (right): (a) and (b) for the isolated nacelle, (c) and (d) for the nacelle and its support inside the wind-tunnel

\section{CAA computations}

\section{Acoustic sources}

In all the CAA simulations, an acoustic source representative of the fan/OGV first interaction mode at the frequency BPF2 is considered. The azimuthal mode is chosen following the well-known theory of Tyler \& Sofrin [24]: $m_{\theta}=n . B-k . V$. Considering the present turbofan characteristics, the first passing mode at BPF2 is an azimuthal mode $m_{\theta}=-4$. Despite the presence of a small distortion in the intake mean velocity field (see figures 4 b) and (d)), no distortion mode is introduced in the computations since no data on the relative amplitudes of these different modes were available. Effects of the mean flow distortion have been investigated in previous paper by Winkler et al.[25] for instance, and at ONERA for both take-off and BSN certification points, see Le Garrec et al.[11] and Daroukh et al.[12]. Only the frequency BPF2 is considered here as it was numerically checked that the frequency BPF1 is cut-off and frequencies BPF3 or more would require too much computational resources for the wind-tunnel case. The source is modelled by

*mainly azimuthal mode $m_{\theta}=1$ 
means of 40 monopoles distributed over a ring with phase shifts, and located inside the nacelle at $90 \%$ of the nacelle inner radius as illustrated in figure 5 .

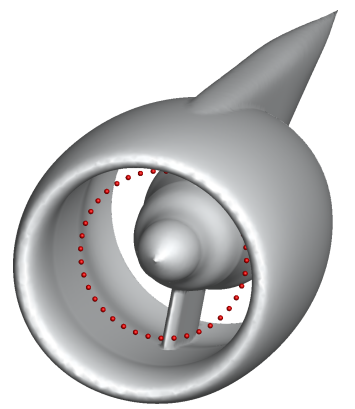

(a)

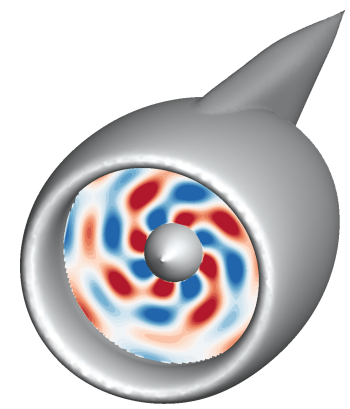

(b)

Fig. 5 Views of (a) monopoles position inside the nacelle and (b) instantaneous pressure field generated in the source plane

\section{Acoustic meshes}

The CAA calculations are carried out with ONERA's sAbrinA_v0 structured CAA solver [3-7] that solves either the full or the linearized Euler's equations, in a conservative and perturbed form. More detailed information about the sAbrinA_v0 solver and its underlying methodology can be found in [26, 27].

The "FF" mesh consists in a single Cartesian grid. A refined mesh zone was defined around the nacelle for acoustic propagation purpose. Inside this zone, the mesh size is constant and enables to resolve the acoustic waves. Outside of the refined mesh zone, a geometrical law with $r=1.05$ is applied in all three directions to increase by a factor 10 the refined mesh size to the computational domain boundaries. The maximum cell size inside the refined mesh zone was calibrated from the target frequency, associated to the BPF2. For this frequency, 12 points per acoustic wavelength were considered in the upstream and downstream directions, the source injection plane being the reference. The mesh size $\Delta x$ associated to the apparent wavelength due to convection effects is then $\Delta x=\lambda .(1 \pm M) / 12$ with $\lambda$ the wavelength at rest and $M$ the Mach number. This gives a total number of nodes of $78 \times 10^{6}$. Crosswise and streamwise cuts of the mesh are presented in figure 6 where the refined mesh zone is delimited by the red lines as well as the different mesh sizes upstream and downstream of the source.

For the "FF_support", "WT_rigid" and "WT_lined" cases, another mesh was designed. Indeed, since the IBM implemented in the $s A b r i n A \_v 0$ solver can currently only be used for objects with rigid walls, it was necessary to design a curvilinear mesh in order to take into account the wind-tunnel walls (which roughly corresponds to a cylinder) and thus being able to impose an impedance boundary condition. The mesh relies on a "O-H" approach and is made of 5 blocks. Moreover, as mentioned in section [II, a Cartesian block has to be introduced in the curvilinear mesh to take into account the nacelle and its support geometries with the IBM. Similarly to the "FF" mesh, a refined mesh zone including the wind-tunnel walls has been defined with the same mesh sizes and the same stretching has been applied in the longitudinal direction. It should be noted that the refined mesh zone does not span the whole longitudinal extent of the wind-tunnel. This was a deliberate choice made in order to reduce the mesh size. With all these considerations, the total number of points is about $695 \times 10^{6}$, which is $\sim 9$ times more than the "FF" mesh because of the larger spatial extent of the mesh necessary to reach the wind-tunnel walls. The final mesh characteristic zones (blocking, refined zone, Cartesian block) are illustrated in figure 7.

For the "WT_lined" case, the impedance model of Delany \& Bazley [28] was used to model the foam panels at the wind-tunnel walls. At BPF2, the absorption coefficient is around $97 \%$ at normal incidence. Furthermore, to ensure a suitable TDIBC (Time Domaine Impedance Boundary Condition), the three-parameter model of Tam \& Auriault[29] has been considered. Several TDIBC models are available in $s A b r i n A \_v 0$ issued from the developments of Escouflaire et al. [30] and Escouflaire [31] based on a Z-transform to traduce the liner characteristics from the frequency domain (where the complex impedance is provided) to the time domain. 


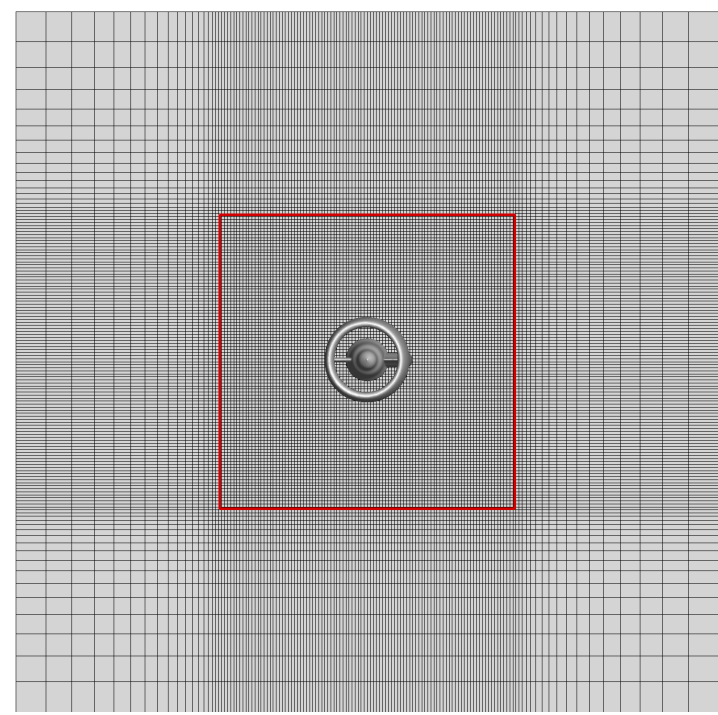

(a)

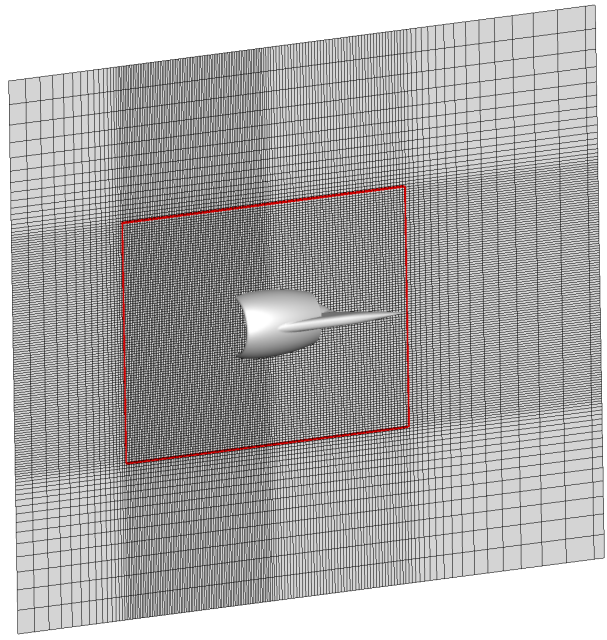

(b)

Fig. 6 Cuts of the CAA Cartesian mesh for the "FF" configuration (a) in the fan stage plane (acoustic source plane) and (b) in a longitudinal plane. The red line delimits the refined mesh zone. For rendering purposes, only one over three mesh points have been displayed.

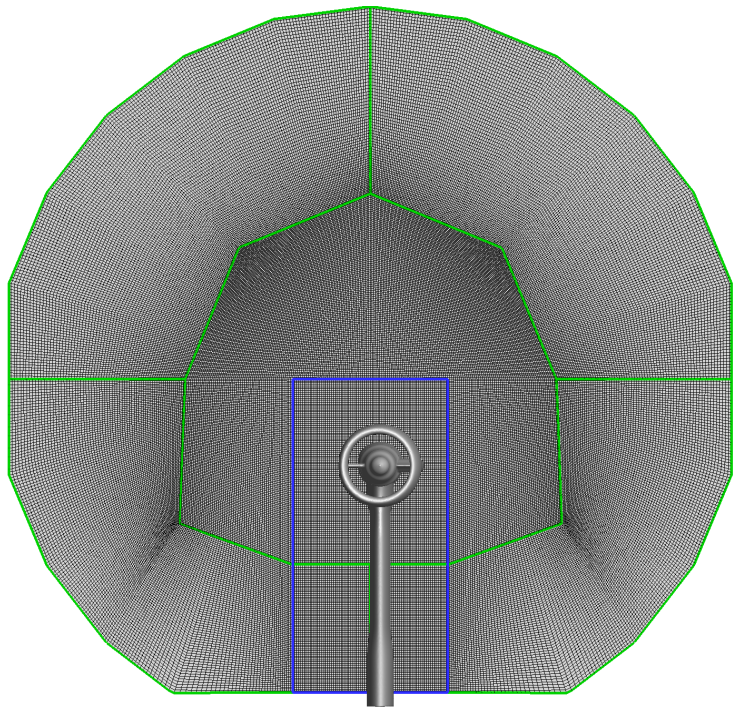

(a)

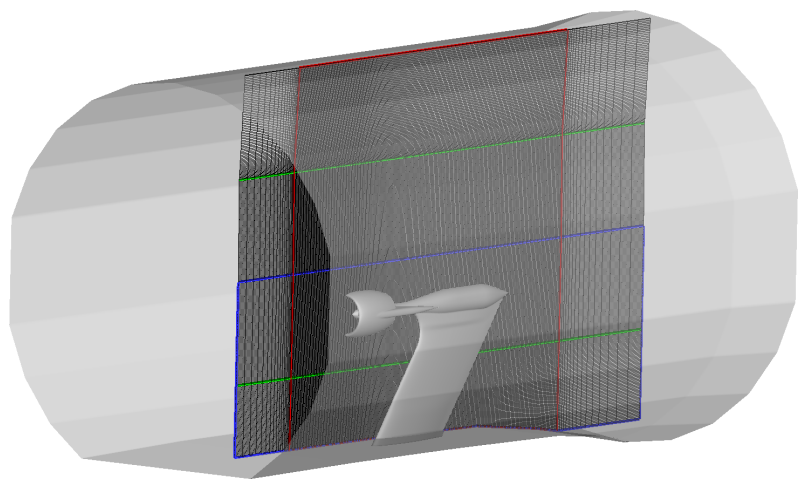

(b)

Fig. 7 Cuts of the CAA curvilinear mesh for the "WT_lined" configuration (a) in the fan stage plane (acoustic source plane) and (b) in a longitudinal mid-nacelle plane. The green lines represent the mesh blocking, the red line the refined mesh zone boundaries and the blue lines the Cartesian mesh block boundaries. For rendering purposes, only one over three mesh points have been displayed.

\section{Acoustic measurements}

The force of numerical simulations compared to experimental tests is the possibility to put probes (antenna) everywhere in the resolved domain without polluting the flow. For the present simulations, once the numerical transient is over, the Fourier transform of the pressure fluctuations is performed on the fly at BPF2 and stored. Several acoustic antennae at different azimuthal angles have been designed for the comparisons between isolated and installed configurations: arc antennae of $2 \mathrm{~m}$ radius centred on the acoustic source plane and longitudinal antennae close to the wind-tunnel walls along the whole longitudinal extent of wind-tunnel refined mesh zone. These antennae are illustrated in figure 8. As these antennae are outside of the refined zone for the "FF" mesh, the direct acoustic solution 
might be inacurrate, so that an indirect solution obtained from a chaining with usual integral method is adopted. To this end, Fourier transform of the pressure fluctuations at BPF2 are stored on prescribed porous surfaces (which are inside the refined mesh zone). These data are then used to feed a Kirchhoff integral formulation (in the frequency domain) implemented in the ONERA in-house code MIA in order to get the extrapolated sound field at the acoustic antennae.

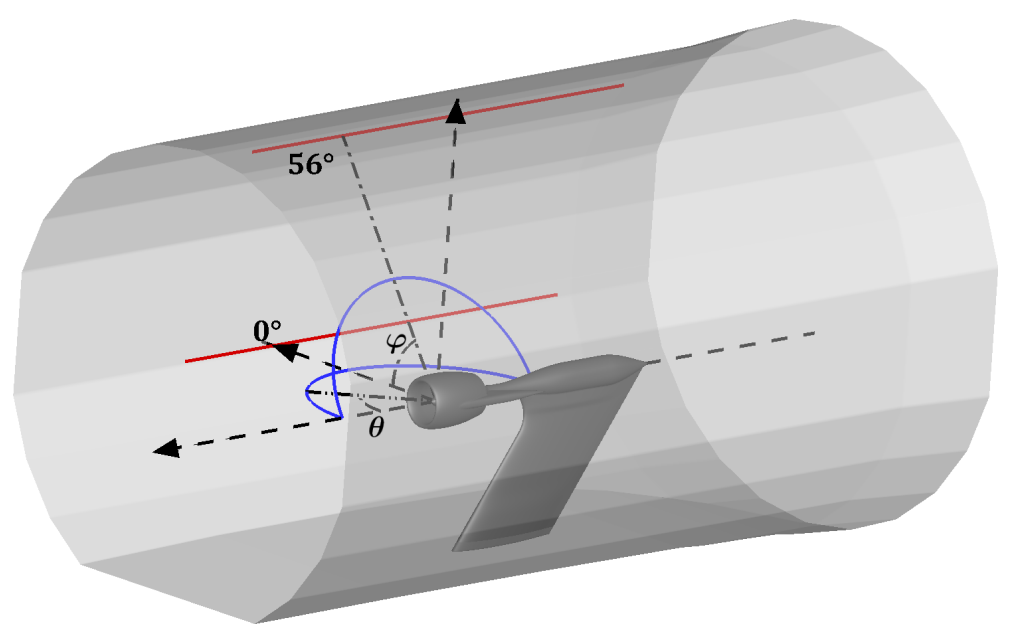

Fig. 8 Antennae definition for the acoustic computations at flyover $\left(0^{\circ}\right)$ and sideline positions $\left(56^{\circ}\right):-$, line antennae; - - arc antennae; at $0^{\circ}$ and $56^{\circ}$

\section{Numerical parameters}

The $s A b r i n A \_v 0$ solver employs high-order, finite difference operators, involving 6th-order spatial derivatives and 10th-order filters, as well as a 3rd-order, compact, Runge-Kutta explicit time-marching scheme. The time step was chosen to get a maximum CFL number of about 0.75 . Simulations are run overt $5 T$ once the convergence is reached (where $\mathrm{T}$ is the period associated to the source frequency) after a numerical transient time of $100 \mathrm{~T}$ for the "FF" simulation and $200 T$ for the "FF_support", "WT_rigid" and "WT_lined" ones. For these three latter cases, a sufficiently long transient period was necessary to ensure that the acoustic waves propagate at least over twice the distance between opposite walls of the wind-tunnel. The "FF" simulation required about 700 elapsed hours (on 112 parallel cores) and the three others about $38 \times 10^{3}$ elapsed hours each (on 1138 parallel cores). These computational times are rather inexpensive compared to computations with curvilinear meshes thanks to the IBM which enables to maximize the time step and thus to minimize the number of iterations for one period of the source. Furthermore, the IBM preprocessing was performed in about $1 \%$ of the overall computational time.

\section{Acoustic results}

In this section, the results obtained for the "FF" and the "WT_lined" simulations are compared. Two other simulations are also included in these comparisons: "FF_support" and "WT_rigid", both performed on the same mesh as the "WT_lined" simulation. This will give a quantitative effect of the impedance condition imposed on the wind-tunnel walls. Since realistic sources would involve unsteady CFD calculations (out of the scope here), simple modal sources with arbitrary levels are injected in the CAA. As previously explained, it is devoted to an evaluation of the confinement effects using dedicated CAA set-up with common prescribed sources.

\section{A. Fluctuating pressure field}

The real part of the fluctuating pressure field associated to the frequency BPF2 for the free-field and confined cases are presented in figures 9, 10 and 11. The same color map levels have been considered. The refined mesh zone boundaries are superimposed in red in these figures. The fast decrease of the pressure waves amplitude due to the mesh stretching is clearly visible. It seems that, in the free-field case, the acoustic radiation is occurring in the upstream 
direction at approximatively $25^{\circ}$ and $45^{\circ}$ from the nacelle axis. These two different directions of radiation can be related to different radial duct modes inside the nacelle. However, given the acoustic source model, there is no control of the acoustic field radial content. The same observation can be made in the downstream direction. This behaviour was expected since interaction modes propagate mainly in the upstream direction whereas the rotor lock mode radiates in the normal plane to the nacelle entrance. Acoustical interferences are also clearly noticeable in the mid nacelle area, where acoustics coming from nacelle's refraction at inlet and outlet interact with each other. The figure 9 also highlights the limited extent of the refined mesh zone of the curvilinear mesh in the upstream direction since in this direction, not all the acoustic radiations reach the wind-tunnel walls before being numerically damped by the stretching. Moreover, the duct mode azimuthal order $m_{\theta}=4$ is recovered as shown in figures 10 and 11. In the downstream direction, the mode structure is clearly altered by the presence of the two internal deviations, contrary to the upstream direction. The latter phenomenum has been widely highlighted in a previous ONERA's study[7].

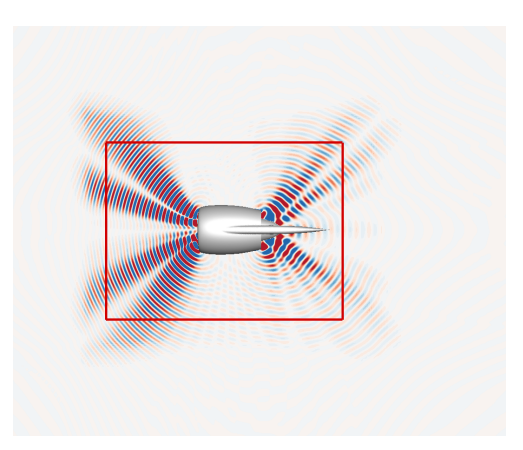

(a)

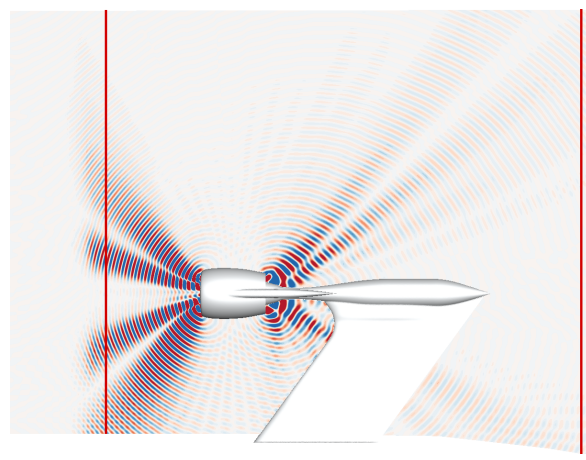

(c)

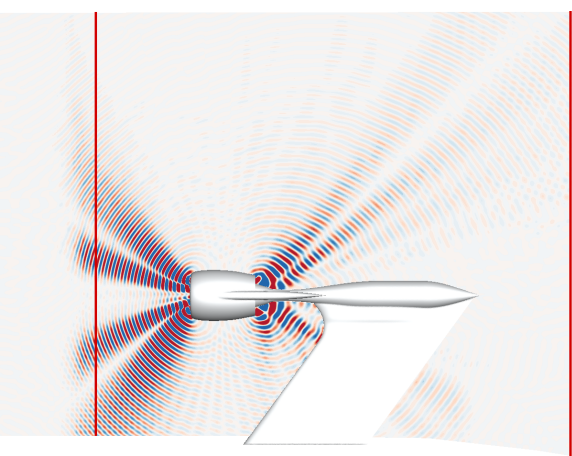

(b)

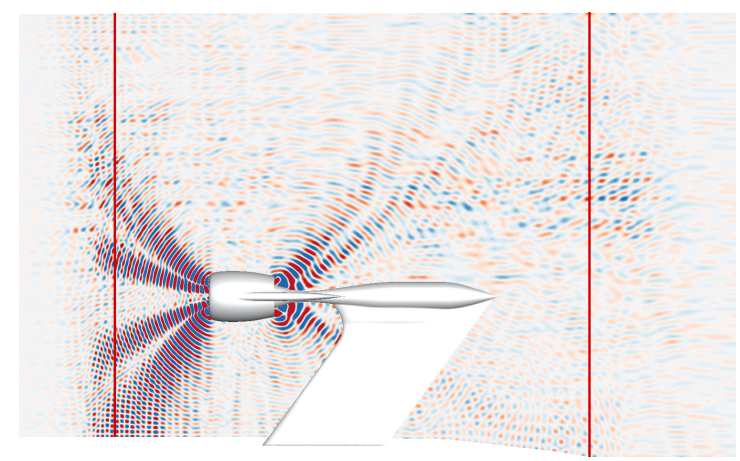

(d)

Fig. 9 Real part of the fluctuating pressure field associated to BPF2 in a vertical longitudinal plane for the (a) "FF", (b) "WT_lined", (c) "FF_support" and (d) "WT_rigid" simulations.

As expected, strong specular reflections occur in the "WT_rigid" simulation (see figures 9 , 10 and 11, the direct acoustic field being disturbed by the backscattered waves especially close to the wind-tunnel walls where the acoustic measurements are likely to be performed. It shows the importance of having a proper acoustic treatment to damp these reflections. The "WT_lined" results are very similar to "FF_support" ones. Indeed, the reflections are greatly attenuated despite some negligible remaining interferences (see figures 10 (b) and (c)). At BPF2, the liner installed on the wind-tunnel walls has an absorption of about $97 \%$ at normal incidence, however this value varies depending on the angle of incidence.

Furthermore, in spite of the presence of the nacelle support, the "WT_lined" pressure field appears to be quite close to the "FF" pressure field. Some small interferences can nevertheless be detected in the downstream positions, especially in the vicinity of the nacelle support, see figures 11 a) and (b). Pressure levels measured on antennae are presented in the following and will give more insight on these observations. 


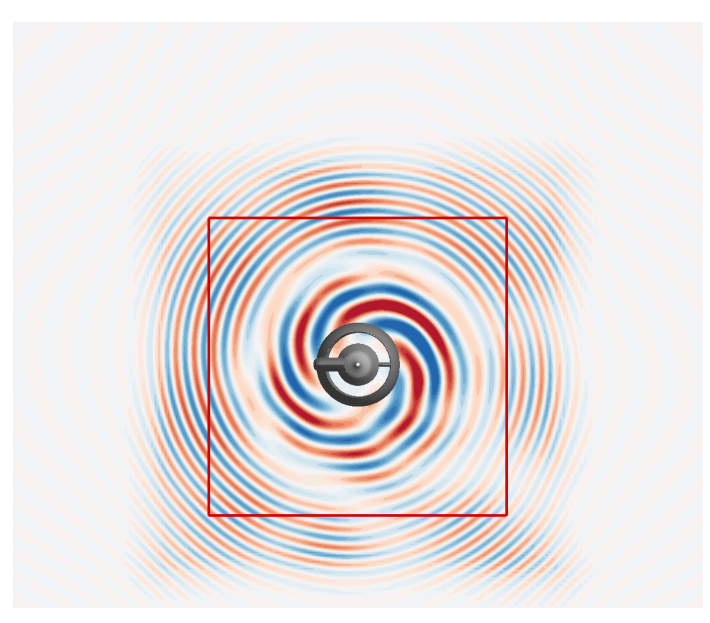

(a)

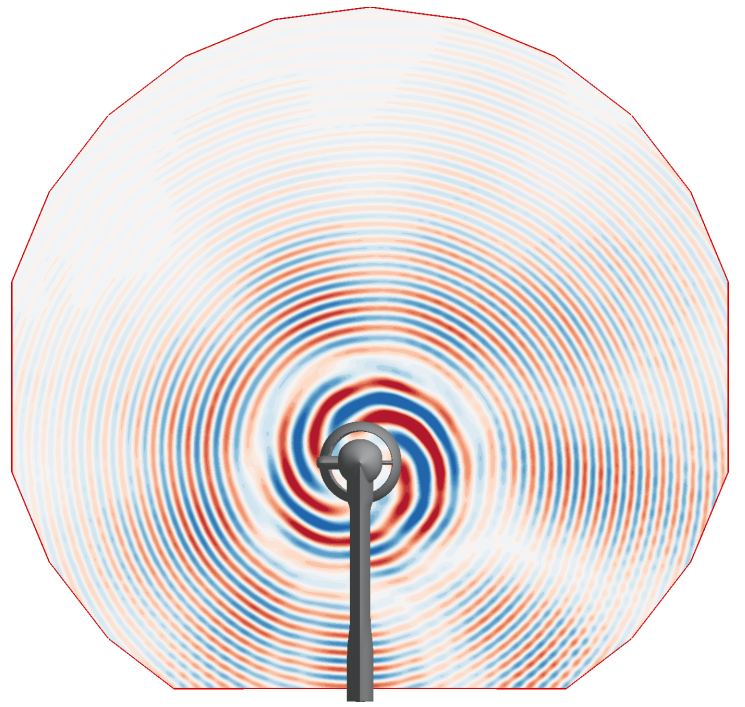

(c)

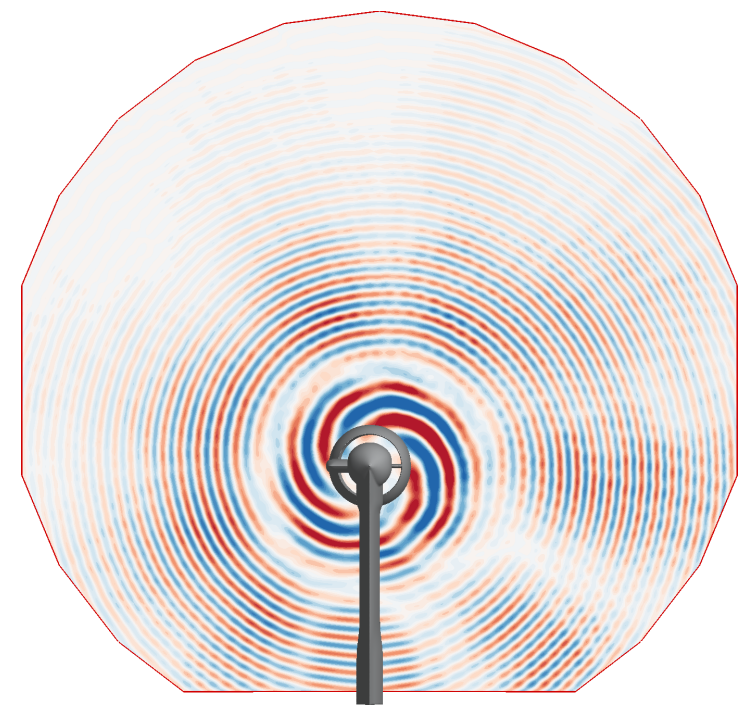

(b)

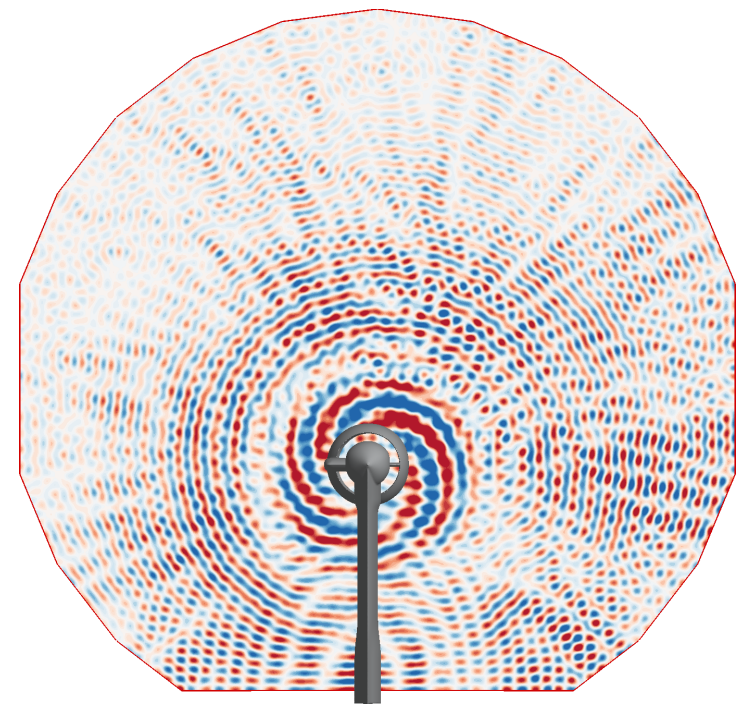

(d)

Fig. 10 Real part of the fluctuating pressure field associated to BPF2 in a upstream crosswise plane for the (a) "FF", (b) "WT_lined", (c) "FF_support" and (d) "WT_rigid" simulations.

\section{B. Quantitative comparisons}

Once the convergence is reached, Fourier transform of the pressure fluctuations at BPF2 is stored on acoustic antennae for the "FF_support", "WT_rigid" and "WT_lined" simulations. For the "FF" case, as explained in section III.C.3. the in-house code MIA based on the Kichhoff integral formulation is employed to evaluate the SPL (Sound Pressure Levels) on these far field antennae.

Evolution of the SPL along the longitudinal acoustic antennae close to the wind-tunnel walls are plotted in figure 12 for two different azimuthal angles. It should be noted that the two antennae are not centred at the same distance from the nacelle axis as the nacelle is not located on the wind-tunnel axis, but a little below (see figures 7), a), 10 and 11 for instance). The flyover antenna is at a shorter distance from the nacelle axis than the other one, so results can not be directly compared. As all the simulations are performed for only one frequency, pronounced interference patterns with large variations of amplitude are visible in these figures.

These comparisons show that the "FF_support" and the "WT_lined" simulations present close results, especially compared to the "WT_rigid" simulation, with some discrepancies, as expected from the results of section IV.A These 


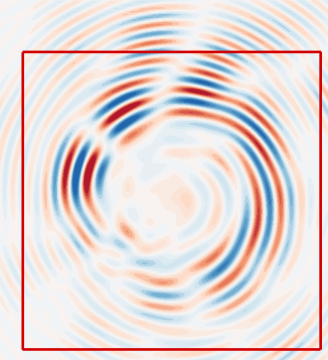

(a)

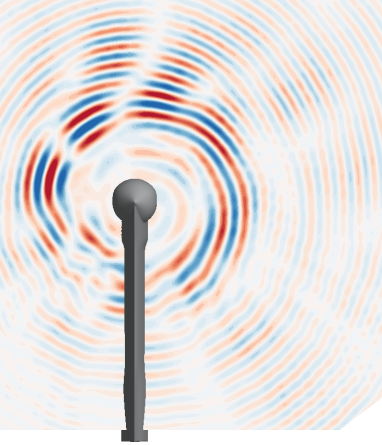

(b)

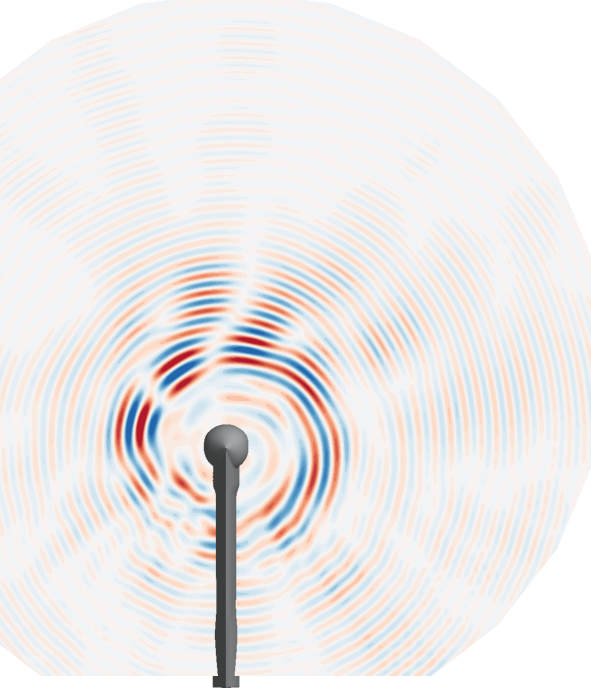

(c)

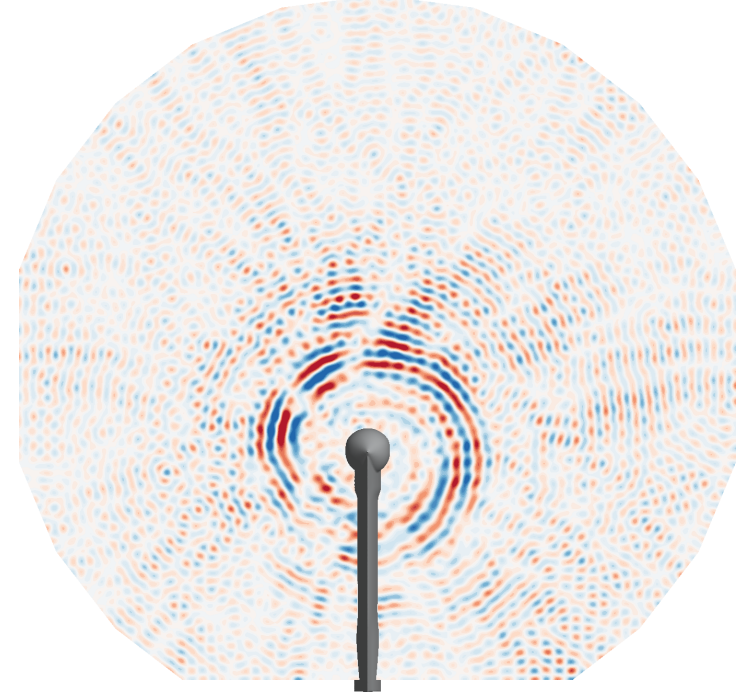

(d)

Fig. 11 Real part of the fluctuating pressure field associated to BPF2 in a downstream crosswise plane for the (a) "FF", (b) "WT_lined", (c) "FF_support" and (d) "WT_rigid" simulations.

discrepancies might come from the variations of the liner absorption coefficient depending on the incidence angle.

From the "FF" and the "WT_lined" results, two observations can be made. Firstly, the two simulations have similar SPL evolutions along the two antennae, particularly in the downstream direction (i.e. for $x-x_{s}>0$ ). This demonstrates that for these positions, the wind-tunnel confinement effect is negligible and that the nacelle support has a limited influence. Secondly, for upstream positions in the nacelle symmetry plane (see figure 12.a)), the "WT_lined" overestimates the "FF" by about 4dB. To explain such overestimation, the SPL close to the nacelle intake are represented in figure 13 , in the same plane as the antenna, for the two simulations. As expected, the "WT_lined" levels are higher, especially close to the nacelle lip on the opposite side of the nacelle pylon. These higher levels might be related to the differences between the two mean flows which can have a great influence on the acoustic duct modes distribution, particularly in presence of mean flow distortion. Further investigations are needed to deal with this physical phenomenum.

As above-mentioned, the longitudinal extent of the mesh for the "confined" simulations is not sufficient to capture the whole acoustic upstream radiation. However, numerical arc antennae centred on the acoustic source plane inside the 


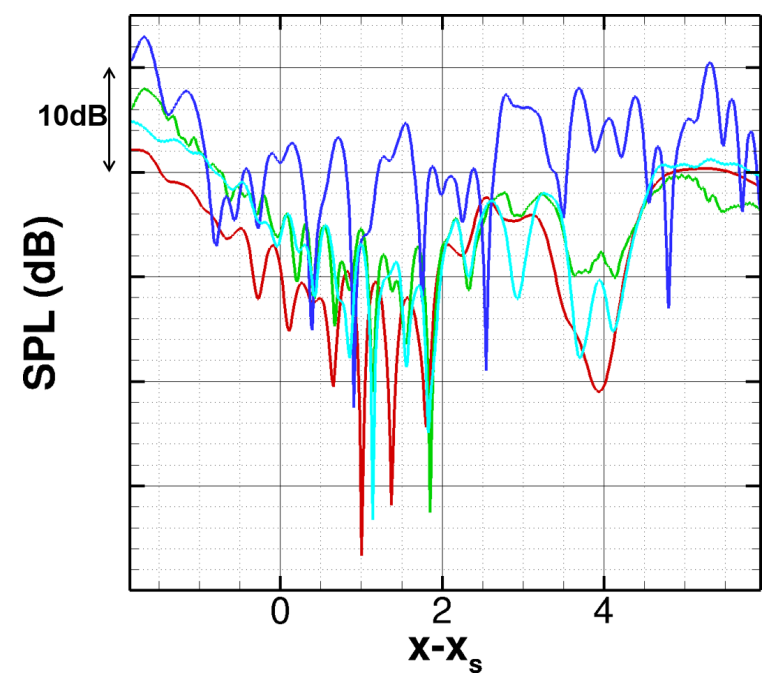

(a)

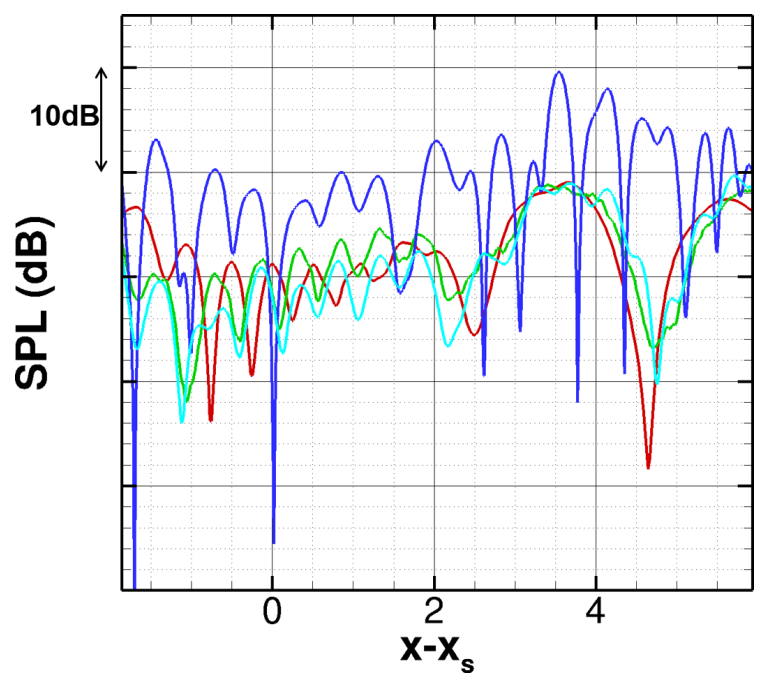

(b)

Fig. 12 Comparisons of the SPL on the longitudinal antenna close to the wind-tunnel walls (a) in the flyover direction and (b) in the sideline direction: "FF", ; "FF_support", ; "WT_rigid", ; "WT_lined",

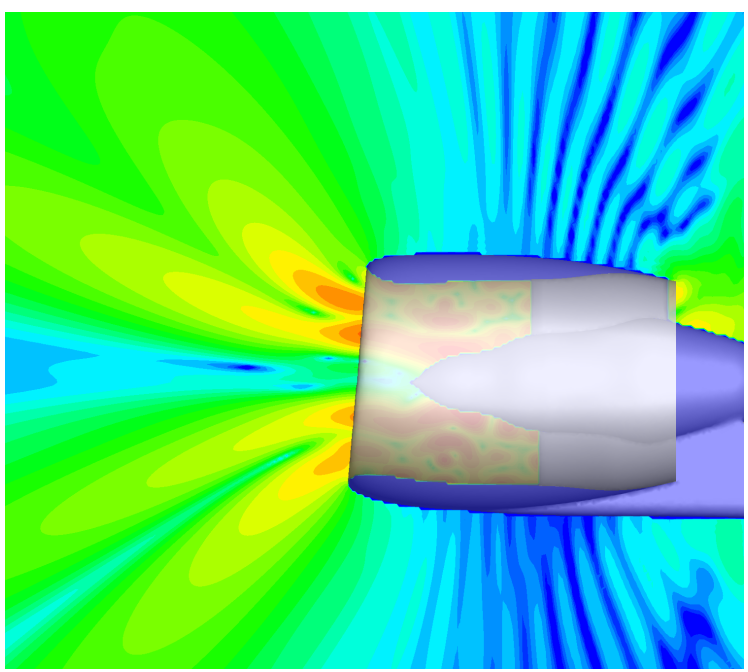

(a)

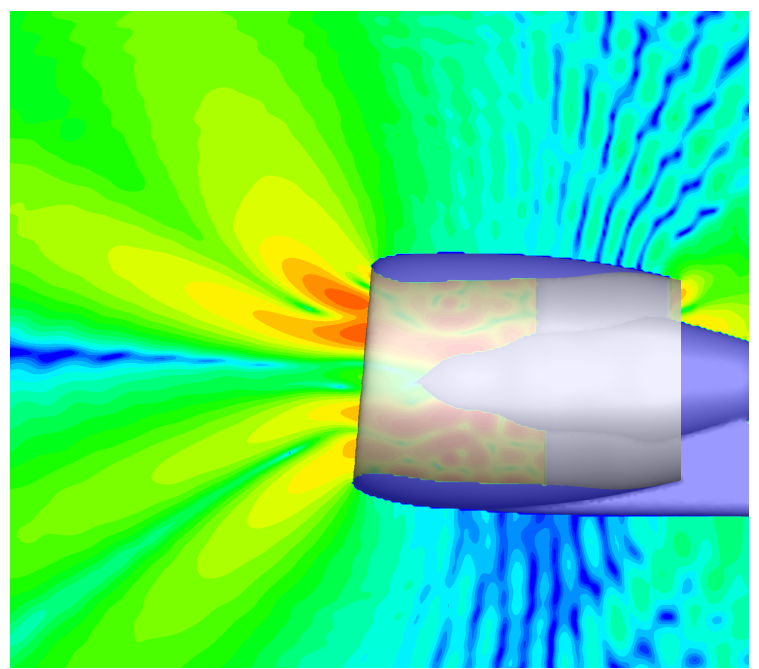

(b)

Fig. 13 Close-up near the nacelle intake of the SPL in the nacelle symmetry plane for (a) the "FF" and (b) the "WT_lined" configurations.

refined mesh zone have been designed. The figure 14 shows the comparison of the SPL on the flyover and sideline arc antennae. In this figure, $\theta=0^{\circ}$ represents the upstream direction, $\theta=180^{\circ}$ the downstream direction and $\theta=90^{\circ}$ is in the acoustic sources plane. Similar remarks can be made concerning the close agreement between the "WT_lined" and the "FF_support" simulations as well as the pronounced interferences present in the "WT_rigid" simulation. As the spherical antenna is closer to the nacelle than the longitudinal antenna ( $2 \mathrm{~m}$ vs. $\sim 4 \mathrm{~m})$, the interferences are less pronounced. The overestimation of the SPL in the upstream direction for the "WT_lined" simulation is once more visible for $\theta$ in the range $\left[50^{\circ} ; 90^{\circ}\right]$. For the other angular positions, the "FF" and "WT_lined" simulations present similar acoustic levels.

In summary, four acoustic simulations with realistic turbofan test bench and wind-tunnel test section geometries and mean flow fields have been performed. The simulations results show that the foam panels installed on the wind-tunnel walls reduce significantly the parasitic reflections and help to recover the free-field configuration acoustic directivity. This makes the numerical acoustic simulation a quite helpful tool for the design of future experimental measurements. 


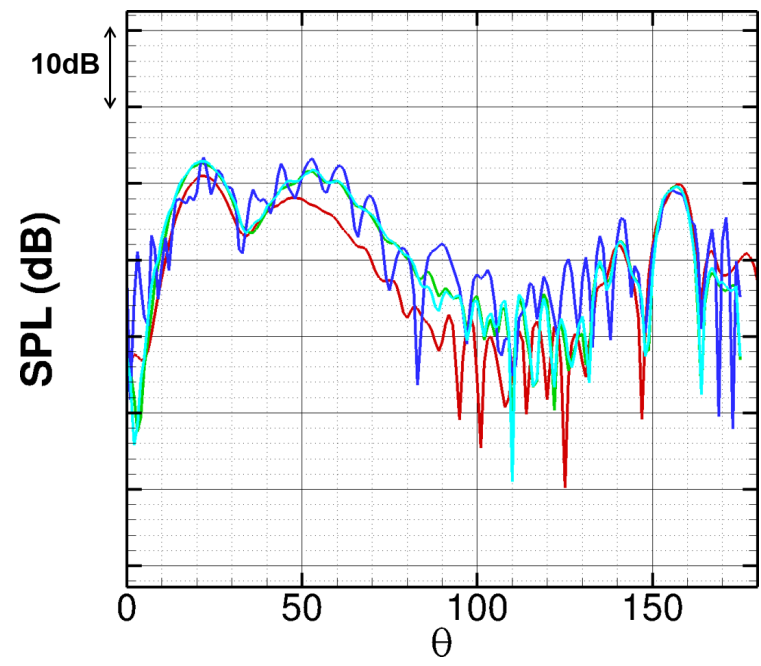

(a)

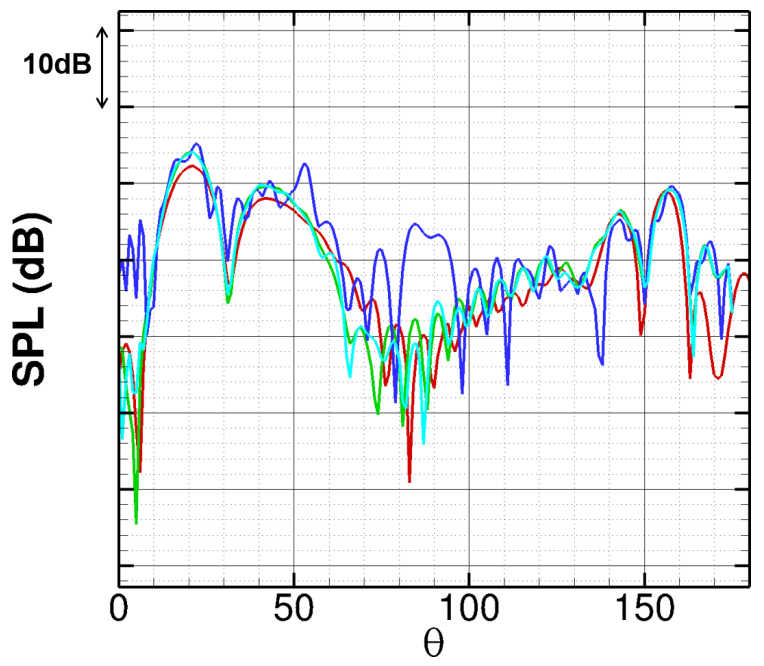

(b)

Fig. 14 Comparisons of the SPL on the $2 \mathrm{~m}$ radius arc antennae centred on the source plane (a) in the flyover direction and (b) in the sideline direction: "FF", _ _ ; "FF_support", __ ; "WT_rigid", _ _ "WT_lined",

\section{Conclusions}

A novel two-step CFD/CAA workflow has been further improved (following previous studies and preliminary results) and successfully applied in order to evaluate the acoustic confinement effects of a turbofan bench at low Mach number inside an aerodynamic wind-tunnel with rigid walls possibly equipped with acoustic liners. This workflow is relying on solid surfaces described by unstructured meshes immersed in 3D Cartesian grids through an efficient Immersed Boundary Method. The present simulations are extended to confined sound propagation with lined walls, thanks to the use of a suited TDIBC implemented in the CAA solver.

Two key simulations have been performed: an isolated nacelle without its support and a nacelle with its support installed in the S1MA wind-tunnel equipped with acoustic foam panels. Two additional simulations (with or without rigid wall) have been included to assess the liner effects (by comparison to a hard wall solution) and its ability to ensure measurements not too far from far-field conditions. The simplified analytical source considered in the CAA is representative of a dominant rotor/stator interaction mode at BPF2. The comparisons confirmed that the confined configuration with acoustic liners on the wall drastically reduces the specular reflections and thus the confinement effects. The acoustic directivity of the isolated nacelle has been retrieved in terms of shape and levels. Some small discrepancies on levels are nonetheless observed and might be related to the different mean flow solutions used for the two acoustic simulations (free-field vs. confined flows). Some further investigations are needed to quantify the latter effect.

Future works could include the full longitudinal extent of the S1MA test section in order to measure the acoustic pressure levels close to the wall in the upstream direction. Moreover, these computations could be easily extended to more realistic sources by considering the acoustic modes related to the inflow distortion as well as a more advanced acoustic source modelling with other interaction modes or a tonal source with higher BPFs. Another possibility of improvement would be to develop the capacity of the implemented IBC method in the sAbrinA_vO solver to be compatible with impedance boundary condition in order to add some liner patches on the nacelle support to be more representative of the future experimental campaign. The numerical tool could also help to determine the optimal position of acoustic antennae for both direct acoustic measurements or beamforming post-processings. Here, all the wind-tunnel walls were equipped with liners: if it is not possible to cover all the walls due to practical set-up restrictions, the numerical tool could help to determine the optimal implementation of the panels to minimize specular reflections.

\section{Acknowledgments}

The project has received funding from the Clean Sky 2 Joint Undertaking under the European Union's Horizon 2020 research and innovation program under grant agreement GA No. 945583 - LPA 2020.

The authors would like to thank Stéphanie Péron, Thomas Renaud and Christophe Benoit for their precious help with the FastS solver and the Cassiopée modules. 


\section{References}

[1] Mincu, D. C., Le Garrec, T., Péron, S., and Terracol, M., "Immersed boundary conditions for high order CAA solversAeroacoustics installation effects assessment," 23rd AIAA/CEAS Aeroacoustics Conference, 2017.

[2] Lorteau, M., Wiart, L., and Kopiev, V., "Numerical study, with experimental validation, of fan noise installation effects in Over-Wing Nacelle configuration using the Immersed Boundary Method," 25th AIAA/CEAS Aeroacoustics Conference, 2019.

[3] Terracol, M., Manoha, E., Herrero, C., Labourasse, E., Redonnet, S., and Sagaut, P., "Hybrid method for airframe noise numerical prediction," Theoretical and Computational Fluid Dynamics, Vol. 19, 2005, pp. 197-227.

[4] Polacsek, C., Desquesnes, G., and Reboul, G., "An equivalent-source model for simulating noise generation in turbofan engines," Journal of Sound and Vibration, Vol. 323, 2009, pp. 697-717.

[5] Redonnet, S., Desquesnes, G., Manoha, E., and Parzini, C., "Numerical study of acoustic installation effects with computational aeroacoustics method," AIAA Journal, Vol. 48, No. 5, 2010, pp. 929-937.

[6] Reboul, G., and Polacsek, C., "Towards Numerical Simulation of Fan Broadband Noise Aft Radiation from Aero-engines," AIAA Journal, Vol. 48, No. 9, 2010, pp. 2038-2048.

[7] Redonnet, S., and Druon, Y., "Computational Aeroacoustics of Realistic Co-Axial Engines," AIAA Journal, Vol. 50, No. 5, 2012, pp. 1029-1046.

[8] Benoit, C., Péron, S., and Landier, S., "Cassiopee: A CFD pre- and post-processing tool," Aerospace Science and Technology, Vol. 45, 2015, pp. $272-283$.

[9] Pilon, A., and Lyrintzis, A., "Integral methods for computational aeroacoustics," 35th Aerospace Sciences Meeting and Exhibit, 1997, p. 20.

[10] Polacsek, C., Burguburu, S., Redonnet, S., and Terracol, M., "Numerical simulation of a fan interaction noise using a hybrid approach,” AIAA Journal, Vol. 44, No. 6, 2006, pp. 1188-1196. doi:10.2514/1.10688.

[11] Le Garrec, T., Polacsek, C., Chelius, A., Daroukh, M., and François, B., "Tone noise predictions of a full-scale UHBR engine at approach condition with inflow distortion effects," 25th AIAA/CEAS Aeroacoustics Conference, Vol. (2606), 2019.

[12] Daroukh, M., Polacsek, C., and Chelius, A., "Shock wave generation and radiation from a turbofan engine under flow distortion," AIAA Journal, Vol. 58, No. 2, 2020, pp. 787-801.

[13] “ONERA S1MA facility," , ???? URL https://www.onera.fr/en/windtunnel/s1ma-continuous-flow-windtunnel-atmospheric-mach-005-mach-1

[14] Cartieri, A., and Hue, D., "Using RANS computations to calculate support interference effects on the Common Research Model," STO workshop: Advanced Wind Tunnel Boundary Simulation, Vol. (STO-MP-AVT-284-07), 2018.

[15] Cartieri, A., Hue, D., Chanzy, Q., and Atinault, O., "Experimental investigations on Common Research Model at ONERAS1MA-Drag prediction workshop numerical results," Journal of Aircraft, Vol. 55, No. 4, 2018.

[16] Nicolas, F., "S1MA wind tunnel new aeroacoustic capability: a traversing microphone array," 24th AIAA/CEAS Aeroacoustics Conference, Vol. (3137), 2018.

[17] Mary, I., "Flexible Aerodynamic Solver Technology in an HPC environment," Maison de la Simulation Seminars, Vol. http://www.maisondelasimulation.fr/seminar/data/201611 slides 1.ppt, 2016.

[18] Péron, S., Renaud, T., Terracol, M., Benoit, C., and Mary, I., “An immersed boundary method for preliminary design aerodynamic studies of complex configurations," 23rd AIAA Computational Fluid Dynamics Conference, Vol. (3623), 2017.

[19] Dandois, J., Mary, I., and Brion, V., "Large-eddy simulation of laminar transonic buffet," Journal of Fluid Mechanics, Vol. 850, 2018, pp. 156-178.

[20] Renaud, T., Benoit, C., Péron, S., Mary, I., and Alferez, N., "Validation of an immersed boundary method for compressible flows," AIAA Scitech 2019 Forum, Vol. (2179), 2019.

[21] Péron, S., Benoit, C., Renaud, T., and Mary, I., "An immersed boundary method on Cartesian adaptive grids for the simulation of compressible flows around arbitrary geometries," Engineering with Computers, 2020. 
[22] Tanguy, G., and Renaud, T., "Flow field assessment of the ONERA L1 Wind Tunnel numerical simulations," AVT-338-RSM Specialists Meeting on Advanced Wind Tunnel Boundary Simulation, 2021.

[23] Spalart, P. R., and Allmaras, S. R., “A one-equation turbulence model for aerodynamic flows," 30th Aerospace Sciences Meeting and Exhibit, Vol. (439), 1992.

[24] Tyler, J., and Sofrin, G., “Axial flow compressor noise studies,” SAE Transactions, Vol. 70, 1962, pp. 309-332.

[25] Winkler, J., Reimann, C. A., Reba, R., Ali, A. A., and Gumke, C., "Inlet and aft tonal noise predictions of a full-scale turbofan engine with bifurcation and inlet distortion," 23rd AIAA/CEAS Aeroacoustics Conference, Vol. (3034), 2017.

[26] Redonnet, S., Manoha, E., and Sagaut, P., "Numerical simulation of propagation of small perturbations interacting with flows and solid bodies," AIAA paper, Vol. (3493), 2001.

[27] Redonnet, S., "Simulation de la propagation acoustique en présence d'écoulements quelconques et de structures solides, par résolution numérique des équations d'Euler," Ph.D. thesis, Université Bordeaux 1, 2001.

[28] Delany, M. E., and Bazley, E. N., "Acoustical properties of fibrous absorbent materials," Applied Acoustics, Vol. 3, 1970, pp. $105-116$.

[29] Tam, C. K. W., and Auriault, L., "Time-domain impedance boundary conditions for computational aeroacoustics," AIAA Journal, Vol. 34, No. 5, 1996, pp. 917-923. doi:10.2514/3.13168, URL https://doi.org/10.2514/3.13168

[30] Escouflaire, M., Redonnet, S., and Aurégan, Y., "Further insights on time-domain impedance boundary condition," 19th AIAA/CEAS Aeroacoustics Conference, 2013, p. 209.

[31] Escouflaire, M., "Theoretical and Numerical Investigation of Time-Domain Impedance Models for Computational AeroAcoustics," Ph.D. thesis, Université du Mans, 2014. 